\title{
Anomalous pressure dependence of the atomic displacements in the relaxor ferroelectric $\mathrm{PbMg}_{1 / 3} \mathrm{Ta}_{2 / 3} \mathrm{O}_{3}$
}

\author{
S. N. Gvasaliya, ${ }^{1, *}$ V. Pomjakushin, ${ }^{1}$ B. Roessli, ${ }^{1}$ Th. Strässle, ${ }^{1,2}$ S. Klotz, ${ }^{2}$ and S. G. Lushnikov ${ }^{3}$ \\ ${ }^{1}$ Laboratory for Neutron Scattering ETHZ \& Paul Scherrer Institut, CH-5232 Villigen PSI, Switzerland \\ ${ }^{2}$ Physique des Milieux Denses, IMPMC, CNRS-UMR 7590, Université Pierre et Marie Curie, F-75252 Paris, France \\ ${ }^{3}$ Ioffe Physical Technical Institute, 26 Politekhnicheskaya, 194021, St. Petersburg, Russia
}

(Received 13 February 2006; published 7 June 2006)

\begin{abstract}
The crystal structure of the $\mathrm{PbMg}_{1 / 3} \mathrm{Ta}_{2 / 3} \mathrm{O}_{3}$ relaxor ferroelectric was studied under hydrostatic pressure up to $\sim 7 \mathrm{GPa}$ by means of powder neutron diffraction. We find a drastic pressure-induced decrease of the lead displacement from the inversion center, which correlates with an increase by $\sim 50 \%$ of the anisotropy of the oxygen temperature factor. The vibrations of the $\mathrm{Mg} / \mathrm{Ta}$ are, in contrast, rather pressure insensitive. We attribute these changes being responsible for the previously reported pressure-induced suppression of the anomalous dielectric permittivity and diffuse scattering in relaxor ferroelectrics.
\end{abstract}

DOI: 10.1103/PhysRevB.73.212102

PACS number(s): 77.80.-e, 61.12.-q, 61.50.Ks, 77.84.Dy

$\mathrm{AB}_{x}^{\prime} \mathrm{B}_{1-x}^{\prime \prime} \mathrm{O}_{3}$ complex perovskites are model compounds for the study of ferroelectricity in disordered crystals. In many of these compounds, ferroelectricity occurs without a structural phase transition, and they are referred as relaxor ferroelectrics, or simply relaxors. ${ }^{1}$ Relaxors have a frequency-dependent peak in the dielectric permittivity, which typically extends over hundreds of degrees Kelvin. In addition, many physical properties of relaxors exhibit anomalies in this temperature range attributed to a so-called diffuse phase transition. Despite numerous studies, the physics of systems exhibiting diffuse phase transitions is not yet well understood.

A well-known example of relaxors is $\mathrm{PbMg}_{1 / 3} \mathrm{Nb}_{2 / 3} \mathrm{O}_{3}$ (PMN). ${ }^{1}$ This crystal has $\mathrm{Pm} \overline{3} \mathrm{~m}$ symmetry; however, displacements of ions from the ideal Wyckoff positions are present and these displacements are temperature dependent. ${ }^{2}$ The first evidence for the appearance of correlations on a short-range scale was found by Burns and $\mathrm{Scott}^{3}$ who observed that the optical refraction index departs from linear law at $T_{d} \sim 620 \mathrm{~K}$, i.e., far above the mean Curie temperature $T_{c m} \sim 270 \mathrm{~K}$. They interpreted this anomaly in terms of growing polarization in small regions of nanometer scale [polar nanoregions (PNR)]. The existence of short-range order was later confirmed by neutron and $\mathrm{x}$-ray diffraction that observed diffuse scattering in the vicinity of Bragg peaks of PMN (Refs. 2, 4 and 9) at temperatures lower than $T_{d}$. A very similar behavior is reported in another well-known relaxor $\mathrm{PbMg}_{1 / 3} \mathrm{Ta}_{2 / 3} \mathrm{O}_{3}$ (PMT). ${ }^{1}$ The departure from linear law in the temperature variation of the optical refraction index of PMT was observed ${ }^{5}$ at $T \sim 570 \mathrm{~K}$. As for PMN, temperaturedependent diffuse scattering ${ }^{6}$ and ionic displacements ${ }^{7}$ were also found in PMT.

In the course of studies of PMN and PMT, a close relationship between the diffuse scattering and the amplitude of the displacements of the ions from the special Wyckoff positions in the perovskite structure has been found. ${ }^{7,8}$ In addition, it was observed recently that the susceptibility of the dynamic (quasi-elastic) component of the diffuse scattering in PMN follows well the peak of the dielectric permittivity. ${ }^{9}$

Most of the studies on relaxor ferroelectrics have concentrated on temperature and electric-field effects. Recently, in- terest in the properties of the relaxors under hydrostatic pressure has aroused. ${ }^{10-17}$ Hydrostatic pressure applied to relaxors usually causes: (i) the suppression of the peak in the dielectric permittivity, as found, e.g., in PMN (Ref. 15) or in $\mathrm{PbIn}_{1 / 3} \mathrm{Nb}_{1 / 2} \mathrm{O}_{3}$ (PIN) ${ }^{11}$ and (ii) the decrease in the intensity of the diffuse scattering as observed also, e.g., in PMN (Ref. 16) and PIN. ${ }^{12}$ However, up to now there exists no attempt to link these two effects to the underlying structural changes in these systems.

Here, we present a detailed structural investigation under pressure, aimed to clarify the microscopic origin for the anomalous pressure effects reported in relaxors. We applied neutron diffraction taking advantage of a better sensitivity on the oxygen position as compared to x-ray studies. The experiments were carried out on PMT, which at ambient pressure, shows a broad frequency-dependent anomaly of the dielectric response with a maximum at a frequency of $10 \mathrm{kHz}$ in the vicinity of $170 \mathrm{~K} .{ }^{1}$ In the entire range of temperatures and applied electric fields, the symmetry of PMT is known to remain cubic (space group $P m \overline{3} m$ ). ${ }^{1,7,18}$

The powder diffraction experiment was carried out at ambient temperature on the multidetector high-resolution powder diffractometer (HRPT) ${ }^{19}$ at the spallation neutron source SINQ (Switzerland). ${ }^{20}$ Measurements were performed with a neutron wavelength of $\lambda=1.494 \AA$. Typical exposure times were $\sim 15 \mathrm{~h}$. The polycrystalline sample of PMT had a volume of $\leq 100 \mathrm{~mm}^{3}$ and was loaded into an encapsulated zero-matrix TiZr gasket ${ }^{21}$ with a deuterated methanolethanol (4:1) mix as a pressure-transmitting medium thus enabling hydrostatic pressure conditions up to the highest investigated pressure of $\sim 7 \mathrm{GPa}$. To apply pressure, the VX3 version of the Paris-Edinburgh press ${ }^{22}$ was used. A detailed description of this opposed-anvil setup is given in Ref. 23. The values of the applied pressure were estimated to better than $\pm 0.2 \mathrm{GPa}$ from the change in the unit cell volume of PMT. To this end, the third-order Birch-Murnaghan equation of state $^{24}$ was used with parameters taken from Ref. 16.

The crystal structure was refined with Fullprof ${ }^{25}$ following a structural model of PMT developed in Ref. 7. This study established that the structure is best described by $\mathrm{Pb}$ ions displaced along the $\langle 110\rangle$ direction from the $\left(\begin{array}{lll}0 & 0 & 0\end{array}\right)$ 
(a)

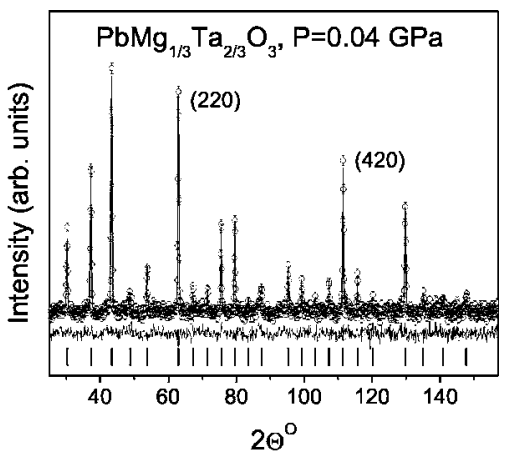

(b)

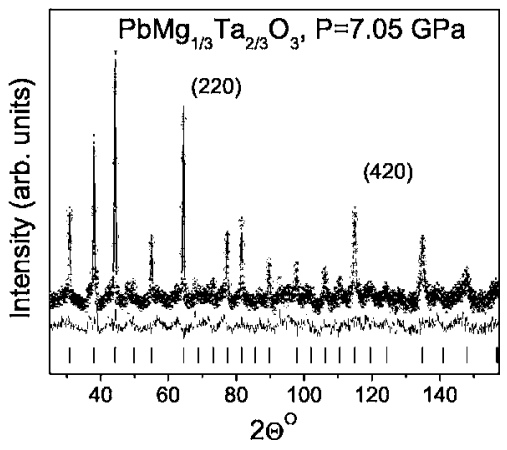

FIG. 1. Neutron powder diffraction patterns of PMT collected at nominal pressures of (a) $P=0.04 \mathrm{GPa}$ and (b) $P=7.05 \mathrm{GPa}$. Observed data points with background originating from the pressure cell is subtracted, calculated profiles and difference curves are shown. The row of ticks corresponds to the calculated positions of diffraction peaks. The peak in the vicinity of $2 \theta=45^{\circ}$, which is not fitted well within our model, is due to scattering from an impurity of pyrochlore (Ref. 7).

special position. The temperature factors of all ions are assumed to be isotropic with the exception of the oxygen ions where anisotropic components are also considered. For the $\mathrm{Mg} / \mathrm{Ta}$ ions, we assume a random occupation of the $\mathrm{Mg} / \mathrm{Ta}$ ions over the $\mathrm{B}$ sites $\left(\begin{array}{lll}0.5 & 0.5 & 0.5\end{array}\right)$ of the PMT perovskite structure according to the stoichiometric ratio. To avoid unwanted correlation between the refined values of the displacement amplitudes and the $\mathrm{B}$ factor of $\mathrm{Pb}$, latter was fixed to a value of $1.14 \AA^{2}$ obtained at $T=300 \mathrm{~K}^{7}$ We note that we cannot rule out a displacement of the $\mathrm{Mg} / \mathrm{Ta}$ or oxygen ions but assert these displacements to be small in comparison to the temperature factors. ${ }^{7}$ The reliability factors for $P=0.04 \mathrm{GPa}$ are $\chi^{2}=1.4, R_{\mathrm{exp}}=2.73, R_{\mathrm{wp}}=3.23, R_{\mathrm{Bragg}}$ $=8.22$, and for $P=7.05 \mathrm{GPa}$, they are $\chi^{2}=1.47, R_{\exp }=1.82$, $R_{\mathrm{wp}}=2.21, R_{\mathrm{Bragg}}=11.1$.

Figure 1 shows representative observed and calculated diffraction patterns obtained for PMT close to ambient and at the highest pressure of nominally $P=7.05 \mathrm{GPa}$. Apart from the trivial shift of all peaks to higher scattering angles related to the contraction of the PMT lattice, the effect of pressure is to modify significantly the relative peak intensities, as seen, for example, on the (220) and (420) reflection. The latter results from the combined effects of the isostructural displacement of the ions within the unit cell and microstrains that broadens Bragg peaks and which may be quantified by a Rietveld analysis of the data. Here we note that sharp superlattice reflections are observed above $\sim 4 \mathrm{GPa}$ in PMN and

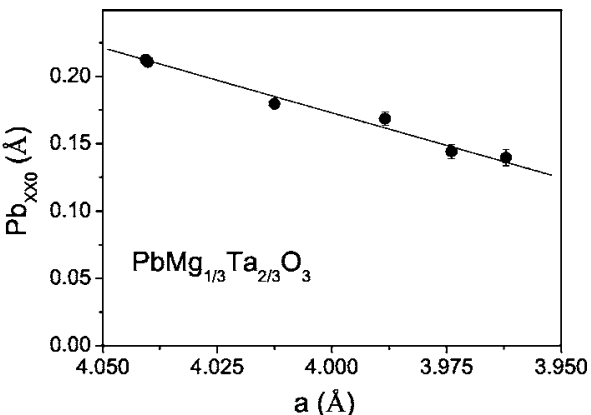

(b)

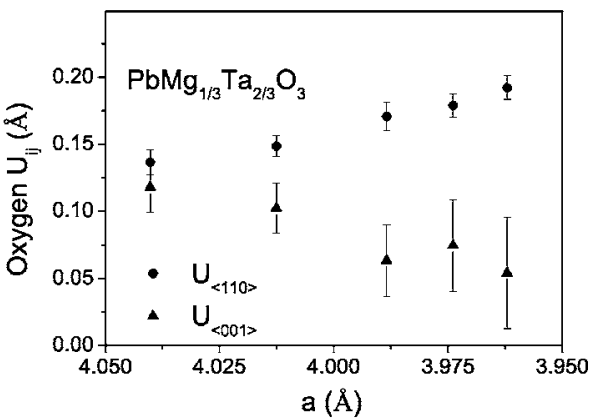

(c)

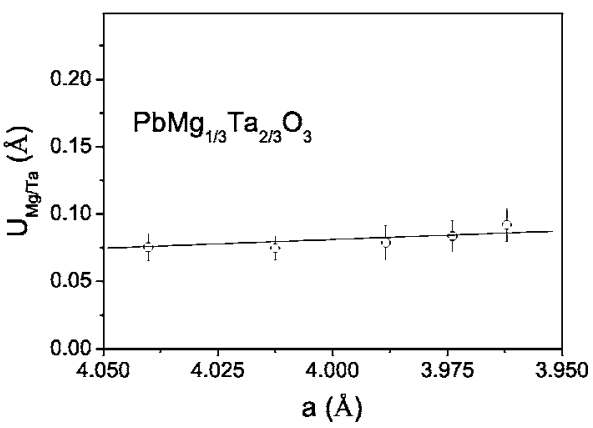

FIG. 2. (a) Dependence of the amplitude of lead displacements $\mathrm{Pb}_{\langle X X 0\rangle}$ in PMT against lattice parameter. Error bars are within the size of the symbols. (b) Root-mean-square displacements of the oxygen ions against lattice parameter: note that the $\langle 110\rangle(\langle 001\rangle)$ direction points toward $\mathrm{Pb}(\mathrm{Mg} / \mathrm{Ta})$. (c) Root-mean-square displacements of the $\mathrm{Mg}$ and $\mathrm{Ta}$ ions against lattice parameter. All graphs have the same scale to emphasize the significant difference in the pressure dependences of these parameters. Values of corresponding pressures can be found in Table I.

$\sim 6 \mathrm{GPa}$ in $\mathrm{PZN}$ at room temperature ${ }^{16,26}$ by $\mathrm{x}$ ray. These reflections are indexed as $(h, k, l) / 2(h, k, l$ - odd) and, hence, correspond to cell doubling. Within the statistical accuracy of the present measurements, these reflections are not observed and the chemical structure of PMT is well described in the $P m \overline{3} m$ space group at all pressures.

Figure 2 shows the evolution of the lead displacements $\left(\mathrm{Pb}_{\langle X X 0\rangle}\right)$ and the root-mean-square (rms) displacements of $\mathrm{Mg} / \mathrm{Ta}$ and oxygen in PMT as a function of the lattice parameter. ${ }^{27}$ These quantities vary approximately linearly with the lattice parameter, however, with opposite slopes and with different rates: e.g., whereas the displacement of $\mathrm{Pb}_{\langle X X 0\rangle}$ decreases by about $0.07 \AA$ as the lattice contracts, the rms of the $\mathrm{Mg} / \mathrm{Ta}$ ions increases by about $0.02 \AA$. It is worth noting that all observed changes in the $\mathrm{Pb}_{\langle X X 0\rangle}$ displacements and 
TABLE I. Pressure, lattice parameter and amplitude of lead displacement of PMT (equation of state with $B=104 \mathrm{GPa}, B^{\prime}=4.7$ taken from Ref. 16).

\begin{tabular}{lcc}
\hline \hline $\begin{array}{l}\text { Pressure } \\
(\mathrm{GPa})\end{array}$ & $\begin{array}{c}\text { Lattice parameter } \\
(\AA)\end{array}$ & $\begin{array}{c}\text { Pb displacement } \\
(\AA)\end{array}$ \\
\hline 0.04 & $4.04019(7)$ & $0.211(3)$ \\
2.28 & $4.01260(7)$ & $0.180(3)$ \\
4.47 & $3.98822(9)$ & $0.169(5)$ \\
5.86 & $3.9738(1)$ & $0.144(5)$ \\
7.05 & $3.9620(2)$ & $0.140(6)$ \\
\hline \hline
\end{tabular}

rms displacements of the other ions (e.g., $\mathrm{Mg} / \mathrm{Ta}, 20 \%$ ) are considerably larger than that of the cell volume of PMT $(\sim 6 \%)$.

Further inspection of Fig. 2 shows that the decrease in the $\mathrm{Pb}_{\langle X X 0\rangle}$ is accompanied by an increase in the anisotropy of the oxygen temperature factor. However, a reliable analysis of the components of the anisotropic temperature factors requires high statistical quality of the data. This is hardly achieved in the present experiment under high pressures and correspondingly small sample volumes. Thus, in order to unambiguously analyze the change in the anisotropy of the oxygen vibrations, we have combined earlier temperaturedependent studies of the PMT structure ${ }^{7}$ with the present results. It turns out that the amplitude of the $\mathrm{Pb}_{\langle X X 0\rangle}$ is a suitable variable common to both the pressure- and temperature-dependent studies. Figure 3 thus shows the components of the oxygen thermal ellipsoid in relation to the amplitude of $\mathrm{Pb}$ displacements. It is clearly seen that the $\langle 110\rangle$ and $\langle 001\rangle$ components of the oxygen temperature factor evolve in the opposite direction; whereas, the component directed along the $\mathrm{Pb}-\mathrm{O}$ bond increases significantly as the amplitude of the $\mathrm{Pb}$ displacements decreases, and the component directed along the $\mathrm{Mg} / \mathrm{Ta}-\mathrm{O}$ bond exhibits a slight decrease. In fact, the latter component of the oxygen temperature factor is the only structural parameter, which has a similar relative change as the PMT cell volume.

The above-evidenced pressure-induced changes of the temperature factors in PMT are, by far, anomalous and may be contrasted to what is expected for a simple solid. In the latter case, the temperature factors are related to the phonons and thus should decrease with increasing pressure at a rate of approximately the average Grüneisen-parameter, that is close to 1 . This implies the relative change of the temperature factors to scale with the change in the unit cell volume. Obviously, the corresponding changes in PMT are in excess to such scaling and even show opposite trends for $\mathrm{Mg} / \mathrm{Ta}$ and for one of the components of the oxygen temperature factors.

Because the perovskite-type materials constitute a significant part of the Earth's mantle, their properties under high pressure are widely studied. However, we are not aware that substantial changes of the atomic temperature factors in these compounds have been reported before, e.g., the temperature factors of the ions in $\mathrm{GdAlO}_{3}$ and $\mathrm{GdFeO}_{3}$ crystals do not change significantly up to pressures of about $8 \mathrm{GPa} .{ }^{28}$ The relative changes of the atomic positions in these materials all

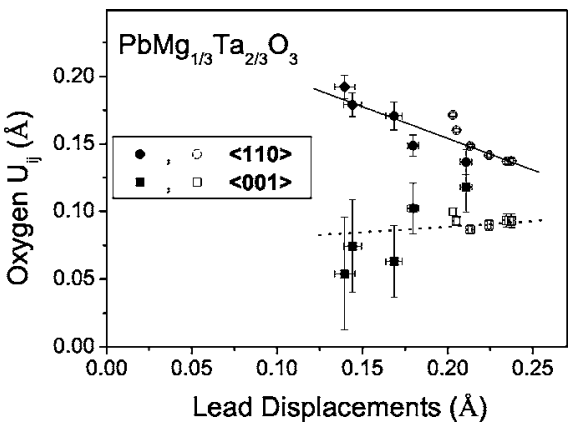

FIG. 3. Components of the oxygen thermal ellipsoid vs the amplitude of lead displacements. Data points shown by open symbols are taken from earlier temperature-dependent studies of the structure of PMT (Ref. 7) at ambient pressure. These points were measured at $T=588,450,300,200,85$, and $1.5 \mathrm{~K}$, correspondingly. We note that smaller $\mathrm{Pb}$ displacements correspond to higher temperatures.

scale well with the changes in the unit cell volume.

On the other hand, it is known that hydrostatic pressure suppresses the ferroelectric properties in perovskites. ${ }^{29}$ For example, at sufficiently high pressures $\mathrm{BaTiO}_{3}$ (Ref. 30) and $\mathrm{KNbO}_{3}$ (Ref. 31) restore the paraelectric cubic structure. ${ }^{32}$ However, even such significant changes are not accompanied by profound changes in the temperature factors. In the case of the tetragonal-to-cubic pressure-induced phase transition of $\mathrm{BaTiO}_{3}$, there is a shift of the Ti ion by $\sim 0.02 \AA$ and the temperature factor of $\mathrm{Ti}$ increases by a factor of 2 , whereas the structure parameters of the other ions remain essentially unchanged. ${ }^{30}$

From the discussion above, we suggest that the pressureinduced changes in the structural parameters of PMT might also be observed in similar relaxors. The most significant of these changes is the decrease of the amplitudes of $\mathrm{Pb}$ displacements and increase of the component of the anisotropic temperature factor of oxygen ions directed toward $\mathrm{Pb}$. On the other hand, the change in the temperature factor of $\mathrm{Mg} / \mathrm{Ta}$ is much less pronounced. From the analysis of the Bragg peak intensities, we cannot infer direct information about the short-range correlations between the displaced ions. However, it is reasonable to expect that as the value of the ionic displacements decreases, the associated short-range order (static and/or dynamic) diminishes, which, in turn, causes (i) the reduction of the diffuse scattering and (ii) the suppression of the dielectric permittivity peak. Thus, our observations give a microscopic explanation for the aforementioned suppressions in lead-containing relaxors, namely, by the anomalous correlated displacements of the lead and oxygen ions. A quantitative model to describe such relationship is a matter of future theoretical and experimental studies. High pressure measurements of the diffuse scattering using single crystals would be highly desirable.

We thank D. Francis (ISIS Facility) for providing us with TiZr gaskets. This work was performed at the spallation neutron source SINQ, Paul Scherrer Institut, Villigen (Switzerland) and was partially supported by RFBR Grant No. 0502-17822 and by Grant of the President No. RF ss1415.2003.2. 
*On leave from Ioffe Physical Technical Institute, 26 Politekhnicheskaya, 194021, St. Petersburg, Russia. Electronic address: severian.gvasaliya@psi.ch

${ }^{1}$ G. A. Smolenskii et al., Ferroelectrics and Related Materials (Gordon and Breach, New York, 1984).

${ }^{2}$ N. de Mathan, E. Husson, G. Calvarin, J. R. Gavarri, A. W. Hewat, and A. Morell, J. Phys.: Condens. Matter 3, 8159 (1991).

${ }^{3}$ G. Burns and B. A. Scott, Solid State Commun. 13, 423 (1973).

${ }^{4}$ S. B. Vakhrushev, B. E. Kvyatkovsky, A. A. Nabereznov, N. M. Okuneva, and B. P. Toperverg, Physica B 156-157, 90 (1989); K. Hirota, Z.-G. Ye, S. Wakimoto, P. M. Gehring, and G. Shirane, Phys. Rev. B 65, 104105 (2002).

${ }^{5}$ O. Y. Korshunov, P. A. Markovin, and R. V. Pisarev, Ferroelectr., Lett. Sect. 13, 137 (1992).

${ }^{6}$ S. N. Gvasaliya, B. Roessli, and S. G. Lushnikov, Europhys. Lett. 63, 303 (2003).

${ }^{7}$ S. N. Gvasaliya, B. Roessli, D. V. Sheptyakov, S. G. Lushnikov, and T. A. Shaplygina, Eur. Phys. J. B 40, 235 (2004).

${ }^{8}$ S. B. Vakhrushev, A. A. Naberezhnov, N. M. Okuneva, and B. N. Savenko, Phys. Solid State 37, 1993 (1995).

${ }^{9}$ S. N. Gvasaliya, S. G. Lushnikov, and B. Roessli, Phys. Rev. B 69, 092105 (2004); S. N. Gvasaliya, B. Roessli, R. A. Cowley, P. Huber, and S. G. Lushnikov, J. Phys.: Condens. Matter 17, 4343 (2005).

${ }^{10}$ G. A. Samara, Phys. Rev. Lett. 77, 314 (1996).

${ }^{11}$ N. Yasuda, H. Ohwa, J. Oohashi, K. Nomura, H. Terauchi, M. Iwata, and Y. Ishibashi, J. Phys. Soc. Jpn. 66, 1920 (1997).

${ }^{12}$ K. Nomura, T. Shingai, S. Ishino, H. Terauchi, N. Yasuda, and H. Ohwa, J. Phys. Soc. Jpn. 68, 39 (1999).

${ }^{13}$ G. A. Samara, E. L. Venturini, and V. H. Schmidt, Appl. Phys. Lett. 76, 1327 (2000).

${ }^{14}$ J. Kreisel, A. M. Glazer, P. Bouvier, and G. Lucazeau, Phys. Rev. B 63, 174106 (2001).

${ }^{15}$ W. Nawrocik, P. Czarnecki, A. Hilczer, and R. Blinc, Phase Transitions 76, 693 (2003).
${ }^{16}$ B. Chaabane, J. Kreisel, B. Dkhil, P. Bouvier, and M. Mezouar, Phys. Rev. Lett. 90, 257601 (2003).

${ }^{17}$ G. A. Samara and E. L. Venturini, Phase Transitions 97, 21 (2006).

${ }^{18}$ Z. G. Lu, C. Flicoteaux, and G. Calvarin, Mater. Res. Bull. 31, 445 (1996).

${ }^{19}$ P. Fischer et al., Physica B 276-278, 146 (2000).

${ }^{20}$ W. E. Fischer, Physica B 234-236, 1202 (1997).

${ }^{21}$ W. G. Marshall and D. J. Francis, J. Appl. Crystallogr. 35, 122 (2002).

${ }^{22}$ J. M. Besson, R. J. Nelmes, G. Hamel, J. S. Loveday, G. Weill, and S. Hull, Physica B 180-181, 907 (1992).

${ }^{23}$ S. Klotz, Th. Strässle, G. Rousse, G. Hamel, and V. Pomjakushin, Appl. Phys. Lett. 86, 031917 (2005).

${ }^{24}$ F. J. Birch, Phys. Rev. 71, 809 (1947); F. D. Murnaghan, Am. J. Math. 49, 235 (1937).

${ }^{25}$ J. Rodríguez-Carvajal, Physica B 192B, 55 (1993).

${ }^{26}$ P. E. Janolin, B. Dkhil, P. Bouvier, J. Kreisel, and P. A. Thomas, Phys. Rev. B 73, 094128 (2006).

${ }^{27}$ The decrease of the lattice parameter is an alternative variable to present the increase of hydrostatic pressure.

${ }^{28}$ N. L. Ross, J. Zhao, and R. J. Angel, J. Solid State Chem. 177, 3768 (2004); J. Solid State Chem. 177, 1276 (2004).

${ }^{29}$ G. A. Samara, T. Sakudo, and K. Yoshimitsu, Phys. Rev. Lett. 35, 1767 (1975); L. G. Liu and W. A. Bassett, Elements, Oxides, and Silicates (Oxford University Press, London, 1986).

${ }^{30}$ S. A. Hayward, S. A. T. Redfern, H. J. Stone, M. G. Tucker, K. R. Whittle, and W. G. Marshall, Z. Kristallogr. 220, 735 (2005).

${ }^{31}$ D. Gourdain, E. Moya, J. C. Chervin, B. Canny, and Ph. Pruzan, Phys. Rev. B 52, 3108 (1995); D. Gourdain, Ph. Pruzan, J. M. Besson, S. Klotz, J. C. Chervin, B. Canny, W. G. Marshall, J. S. Loveday, and M. Hanfland, ibid. 65, 054104 (2002).

${ }^{32} \mathrm{~A}$ recent study (Ref. 33) on $\mathrm{PbTiO}_{3}$ suggests a lowering of the initial symmetry $\mathrm{Pm} \overline{3} \mathrm{~m}$ above a critical pressure.

${ }^{33}$ I. A. Kornev, L. Bellaiche, P. Bouvier, P.-E. Janolin, B. Dkhil, and J. Kreisel, Phys. Rev. Lett. 95, 196804 (2005). 